C-A/AP/\#183

December 2004

\title{
Real and Modeled Effect of Booster Extraction Equipment on Dispersion
}

\author{
V. Schoefer, L. Ahrens, K. Brown
}

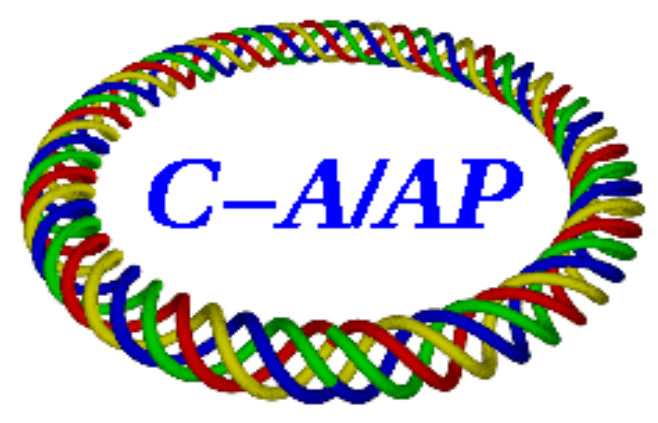

Collider-Accelerator Department Brookhaven National Laboratory Upton, NY 11973 
December 9, 2004

\title{
Real and Modeled Effect of Booster Extraction Equipment on Dispersion
}

\author{
V. Schoefer, L. Ahrens, K. Brown
}

\begin{abstract}
A study was conducted to measure the effect of the Booster extraction equipment on the dispersion function of the Booster when configured for polarized proton acceleration. This study had two primary goals. The first was to verify that the current Booster model accurately predicts the Booster dispersion function and the change in that function caused by the Booster fast extraction 4-bump. The second was to model the effect of the F3 kicker pulse on the dispersion at the F6 septum. The study was motivated in part by a need to provide more accurate Twiss parameter starting values to the BTA model, which begins at the upstream end of the F6 septum.

In order to measure the effect of the extraction bumps on the dispersion, the dispersion was measured at two points in the Booster cycle: at the start of extraction (before the extraction bumps fire), and then again about $3 \mathrm{~ms}$ later at the peak of the extraction bumps when the F3 kicker would ordinarily pulse. The F3 kicker itself did not fire during this observation. These two scenarios were then modeled and the agreement with observation was found to be good.

The experimental measurements of the beam were made using an iron beam probe $(\mathrm{Z}=$ 20 ), which was the most readily available species. The transfer and extraction magnets were set to polarized proton AGS injection values (corresponding to $\mathrm{G} \gamma=4.5$ ). The models were run using MAD8.

To model the effect of the F3 kicker, a new model was constructed consisting only of the elements between the F3 kicker and the F6 septum (inclusive of F3). This model was provided with the Twiss parameter values produced by the model of the full Booster and run with several values for the strength of the F3 magnet. The effect on the dispersion at F6 was found to be small, causing a change of approximately $0.004 \mathrm{~m} / \mathrm{mrad}$ kick.
\end{abstract}

\section{Introduction}

The Booster fast extraction process into the Booster to AGS (BTA) transfer line consists of three major elements: the extraction bump, the F3 kicker and the F6 septum.

The extraction bump consists of a series of four "backlegs". These backlegs are additional windings around the F2, F4, F7 and A1 main dipole magnets. During the extraction process, the four backlegs are pulsed and create a closed orbit distortion in both position and angle centered on the F6 septum. The time dependence of the pulses are half-sine waves with a base width of approximately $5 \mathrm{~ms}$. Further treatment of the Booster extraction equipment can be found in [1] and [2].

At the peak of the extraction bump the F3 kicker fires. The F3 is a kicker magnet consisting of four modules which, when fired, moves the trajectory of the circulating beam across the F6 septum and into BTA. All of the beam in the Booster is extracted in one turn.

The upstream end of the F6 septum is the "hand-off point" between the Booster model and that of BTA. Consequently it appears only as a place-holder (a "marker") in the Booster model. 
There is no PUE at the F6 location owing to the size of the septum itself. As such, the dispersion at that point could not be experimentally verified.

\section{MODELED AND EXPERIMENTAL DATA}

The following parameters were supplied to the MAD8 model of the Booster. These parameters correspond to the flattop of a machine configured for polarized proton acceleration $(\mathrm{G} \gamma$ $=4.5)$ :

\begin{tabular}{|c|c|c|c|c|}
\hline Parameter & $\begin{array}{l}\text { Variable } \\
\text { name } \\
\end{array}$ & \multicolumn{2}{|c|}{ Value* } & $\underline{\text { Units }}$ \\
\hline Main dipole current & IDIPO & \multirow{2}{*}{\multicolumn{2}{|c|}{2178.464}} & $\bar{A}$ \\
\hline Trim quadrupole currents: & & & & \\
\hline Horizontal & IQHC & \multicolumn{2}{|c|}{325.231} & A \\
\hline Vertical & IQVC & \multicolumn{2}{|c|}{371.184} & A \\
\hline B dot & BDOT & \multicolumn{2}{|c|}{5.1} & $\mathrm{~T} / \mathrm{s}$ \\
\hline Bump currents: & & Bump off & Bump on & \\
\hline $\mathrm{F} 2$ & IF2 & 0 & 221.79 & A \\
\hline F4 & IF4 & 0 & 388.86 & A \\
\hline F7 & IF7 & 0 & 499.76 & $A$ \\
\hline $\mathrm{A} 1$ & IA1 & 0 & 754.76 & A \\
\hline
\end{tabular}

Table 1: MAD8 model parameters

*All data retrieved from archives comes from the June 18, 2004 User 4 shift 3 timed archive.

Three separate data sets were produced with these parameters. The first of these, which we call the "off bump" case, set the fast extraction four-bump magnet windings (F2, F4, F7, A1) to zero current. The second of these (the "on bump" case) was produced with the four-bump magnet currents set to their archived setpoint values. Orbit and dispersion data were recorded for both of these configurations.

The third data set was produced with a model consisting only of the Booster line between the F3 kicker magnet and the upstream end of the F6 septum, using the Twiss parameters generated by the "on bump" case as starting values at the F3. The kick at the F3 was then varied over a range from zero through the normal operating range (near $5 \mathrm{mrad}$ for polarized protons) and the orbit and dispersion at the mouth of the F6 calculated.

The model treats the four backleg windings as error fields on the main dipole magnets F2, F4, F7 and A1.

\section{Effect of extraction bump}

Orbits

The orbit data is reproduced below in Figs. 1 (on bump), 2 (off bump) and 3 (on bump off bump). The error bars represent the standard deviation of repeated measurements made over several Booster cycles. Shown below in Table 2 are the r.m.s. differences between the model and the observed data.

Machine errors (i.e. the deviations of the real orbit in the off bump case from zero) are not accounted for in the model and this is expected to degrade the fit of the model to the raw orbit data. The difference orbit, however, should be largely devoid of error harmonics since one can 
expect that they are subtracted out. It should be noted that the tune shift caused by the extraction bump is of order $10^{-3}$ and thus does not significantly affect the strength of the orbit harmonic errors. Any false offsets in the PUEs are also removed by taking the difference.

The r.m.s deviation from the model is not, however, significantly improved with the raw orbit subtracted. The deviation looks to be systematic. The actual currents in the magnets were not recorded at the time of the study and it is probable that the values retrieved from the archives do not quite represent the actual situation, thus degrading the accuracy of the modeled bump.

\section{Dispersion}

Shown below are the resulting dispersion functions (modeled and observed) for the "on bump" (Fig. 4), "off bump" (Fig. 5), and difference data (Fig. 6). Error bars are derived from the error bars of the measured orbits. The r.m.s. differences in dispersion between the model and the observations are summarized below in Table 2. The difference data in Fig. 6 is the least challenging to the system since it only requires the model to be right "at the margin". The full set of MAD output data for the F3 and F6 for the on and off bump cases are found in Tables 4 and 5.

The model predicts that the dispersion at the F6 septum due to the extraction bumps is $2.389 \mathrm{~m}$, where the dispersion with no bump is $2.770 \mathrm{~m}$, a change of about $13 \%$.

\begin{tabular}{|c|c|c|c|c|}
\hline & \multicolumn{2}{|c|}{ Orbit $(\mathrm{mm})$} & \multicolumn{2}{|c|}{ Dispersion (m) } \\
\hline & Beam r.m.s & R.m.s diff. from model & Beam r.m.s & R.m.s diff. from model \\
\hline On bump & 7.13 & 5.50 & 2.21 & 0.20 \\
\hline Off bump & 3.48 & 3.48 & 2.24 & 0.25 \\
\hline On-Off bump & 5.99 & 4.96 & 0.20 & 0.08 \\
\hline
\end{tabular}

Table 2: Accuracy of the MAD model

\begin{tabular}{|l|cc|cc|}
\cline { 2 - 5 } \multicolumn{1}{c|}{} & \multicolumn{2}{c|}{ Dispersion $(\mathrm{m})$} & \multicolumn{2}{c|}{ Dispersion' } \\
\cline { 2 - 4 } & Bump on & Bump off & Bump on & Bump off \\
\hline F3 Kicker & 2.094 & 2.019 & 0.376 & 0.408 \\
F6 Septum & 2.389 & 2.770 & -0.380 & -0.436 \\
\hline
\end{tabular}

Table 3: Modeled D, D'

\section{Effect of F3 kicker}

The modeled change in dispersion caused by F3 is small, approximately $0.004 \mathrm{~m} / \mathrm{mrad}$ kick from the F3, which is nearly two orders of magnitude smaller than the effect of the bumps themselves. The expression for the dispersion at F6 as a function of the kick at F3, with the extraction bumps on (from linear least squares regression on the modeled points) is:

$$
\mathrm{D}=0.003825 \theta+2.389
$$

Here $\mathrm{D}$ is the horizontal dispersion at $\mathrm{F} 6$ in meters, and $\theta$ is the strength of the $\mathrm{F} 3$ kick in milliradians.

As was mentioned, the model's predictions about dispersion at F6 could not be experimentally verified owing to the lack of a PUE at that location. The predicted orbit data, however, agrees with the parameters in the Booster Design Manual [3], showing an orbit 
distortion of about $45 \mathrm{~mm}$ for a $5 \mathrm{mrad}$ kick at F3, where the Manual predicts $50 \mathrm{~mm}$ for the same kick.

\begin{tabular}{|c|c|c|c|c|c|c|c|c|c|c|c|c|c|c|c|}
\hline & AlphaX & BetaX & MuX & Dx & DprimeX & AlphaY & BetaY & MuY & Dy & DprimeY & $x$ & $\mathrm{px}$ & $y$ & Py & s \\
\hline On bump & -1.260 & 7.155 & 1.902 & 2.094 & $\begin{array}{l}0.376 \\
\end{array}$ & $\begin{array}{ll} & 1.273 \\
\end{array}$ & 7.529 & 1.846 & 0.000 & 0.000 & -0.012 & -0.002 & 0.000 & 0.000 & 78.964 \\
\hline Off bump & -1.274 & 7.226 & 1.902 & 2.019 & 0.400 & 1.260 & 7.439 & 1.845 & 0.000 & 0.000 & 0.000 & 0.000 & 0.000 & 0.000 & 78.964 \\
\hline
\end{tabular}

Table 4: MAD TWISS output for the F3 kicker

\begin{tabular}{|l|r|l|l|l|l|l|l|l|l|l|l|l|l|l|l|}
\cline { 2 - 10 } \multicolumn{1}{c|}{} & AlphaX & BetaX & MuX & Dx & DprimeX & AlphaY & BetaY & MuY & Dy & DprimeY X & px & y & Py & s \\
\hline On bump & 1.806 & 12.107 & 2.132 & 2.389 & -0.380 & -0.695 & 4.242 & 2.134 & 0.000 & 0.000 & 0.017 & 0.000 & 0.000 & 0.000 & 90.063 \\
\hline Off bump & 1.800 & 12.024 & 2.131 & 2.770 & -0.428 & -0.701 & 4.286 & 2.134 & 0.000 & 0.000 & 0.000 & 0.000 & 0.000 & 0.000 & 90.063 \\
\hline \multicolumn{8}{c|}{ Table 5: MAD TWISS output for the F6 Septum } &
\end{tabular}



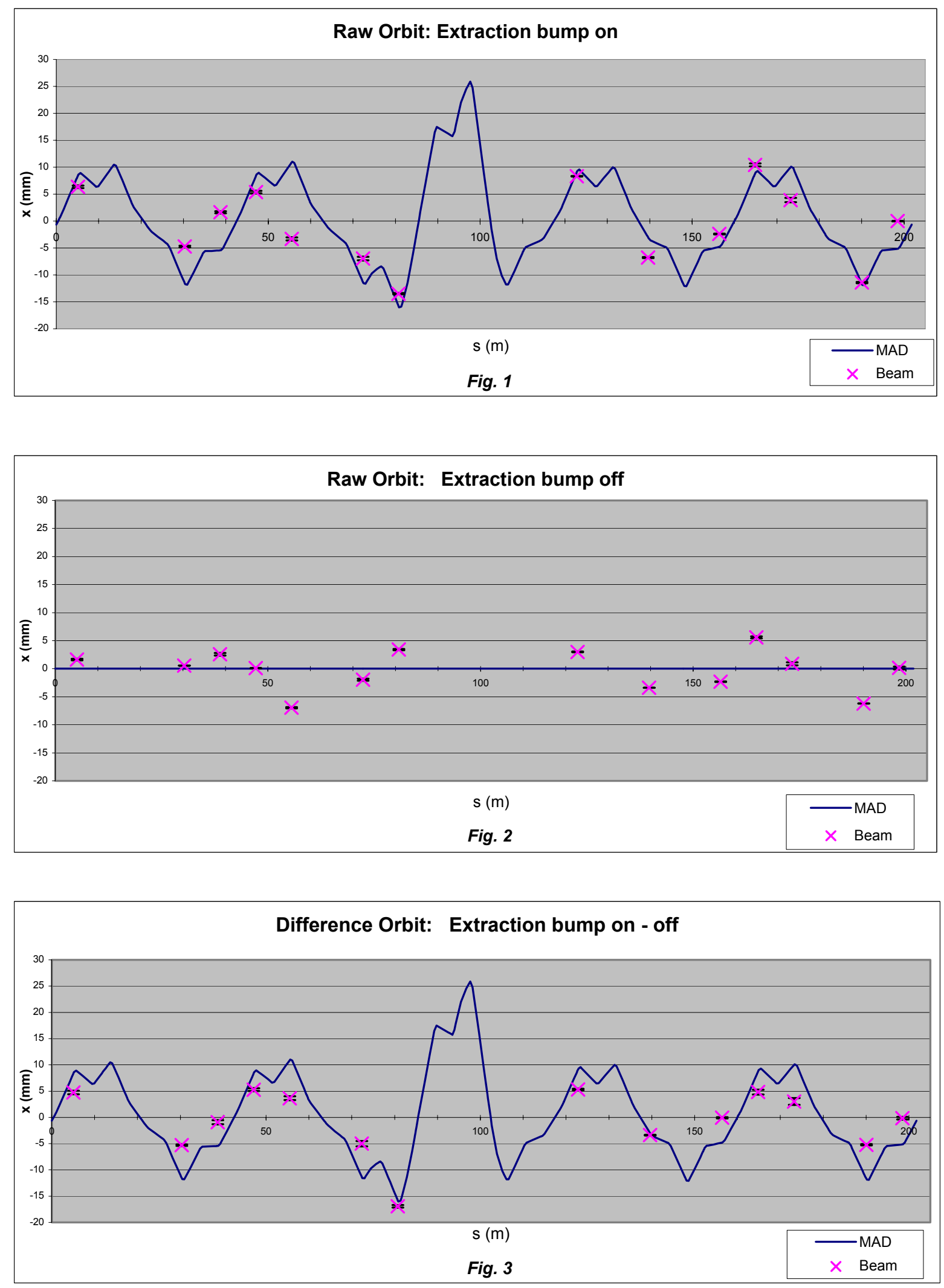

Notes: $\quad$-All values of $s$ are measured from the start of the $D$ superperiod -The F6 septum is at $90.1 \mathrm{~m}$. 

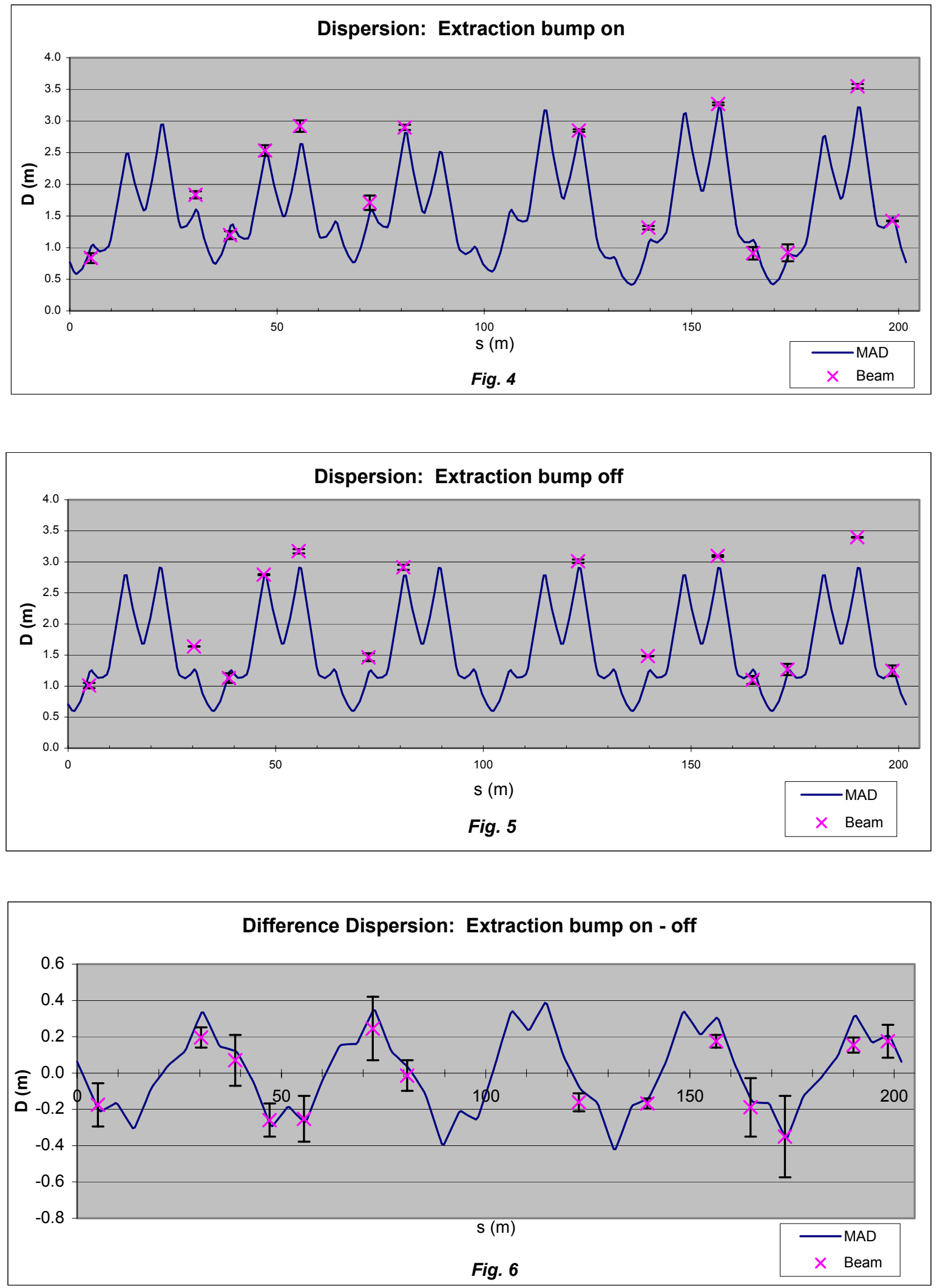

Notes: $\quad$-All values of $s$ are measured from the start of the $D$ superperiod -The F6 septum is at $90.1 \mathrm{~m}$. 


\section{References}

[1] S. Y. Lee, "Tolerance of Beam Extraction Elements for AGS Booster", Booster Technical Note No. 154, December 15, 1989.

[2] A. Luccio, "Bumps in the AGS Booster", Booster Technical Note No. 189, March 11, 1991.

[3] Booster Design Manual (Brookhaven National Laboratory, Upton, NY, 1986).

[4] K. Brown, W. van Asselt, W. Meng, "A high precision model of Booster Tune Control”, C-A/AP Note No. 220, February 10, 1993. 